\title{
Prevalence of anemia and iron deficiency anemia in Chinese pregnant women (IRON WOMEN): a national cross-sectional survey
}

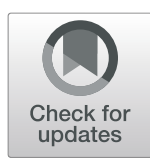

Jing Tan ${ }^{1}$, Guolin He${ }^{2}$, Yana Qi ${ }^{1}$, Hongmei Yang ${ }^{2}$, Yiquan Xiong ${ }^{1}$, Chunrong Liu', Wen Wang ${ }^{1}$, Kang Zou', Andy H. Lee ${ }^{3}$, Xin Sun ${ }^{1}$ and Xinghui Liu ${ }^{2 *}$

\begin{abstract}
Background: The current evidence about anemia and iron deficiency anemia (IDA) during pregnancy remains elusive in China. The purpose of this study is to investigate the prevalence of anemia and IDA and their risk factors in Chinese pregnant women.

Methods: A nationwide cross-sectional survey of pregnant women was conducted during their antenatal visits. Using a multi-stage sampling method, 24 hospitals from 16 provinces across China were selected. Structured questionnaires were administered to collect information from participants and to extract clinical data from electronic medical records. Mixed-effects logistic regression models were performed to determine the risk factors associated with anemia and IDA.

Results: In total, 12,403 pregnant women were enrolled, including 1018 (8.2\%) at the first trimester, 3487 (28.1\%) at the second, and $7898(63.7 \%)$ at the third. Overall, 19.8\% of women were diagnosed with anemia and 13.9\% were diagnosed with IDA. The prevalence of anemia and IDA varied among regions and increased by gestational month, peaking at the eighth gestational month (24.0\% for anemia and 17.8\% for IDA). Pregnant women at advanced stage of gestation, non-local residents, multiple gestations, multiparity, pre-pregnancy underweight, and those experiencing severe nausea or vomiting during pregnancy, were associated with higher risks of anemia and IDA.
\end{abstract}

Conclusions: The prevalence of anemia and IDA during pregnancy are similar to those from developed countries and vary across regions in China.

Keywords: Anemia, Iron deficiency anemia, Prevalence, Risk factors, Cross-sectional survey

\section{Background}

Anemia is the most common pregnancy comorbidity [1, 2]. A systematic review of evidence from 107 countries suggested that the prevalence of anemia among pregnant women was $38 \%$ (95\% CI 33-43\%), impacting approximately 32 million individuals [3], of whom about $75 \%$ were manifested with iron deficiency [4]. The prevalence

\footnotetext{
* Correspondence: xinghuiliu@163.com

${ }^{2}$ Department of Obstetrics and Gynecology, and Key Laboratory of Birth Defects and Related Diseases of Women and Children (Sichuan University), Ministry of Education; West China Second University Hospital, Sichuan University, No.37 Guo Xue Xiang, Chengdu, China

Full list of author information is available at the end of the article
}

of iron-deficient anemia (IDA), the major type of anemia, appears to vary across regions, from $3 \%$ in Europe [5] to over $50 \%$ in Africa [6, 7].

Anemia and IDA can lead to adverse health outcomes for both mothers and their offspring, including infections, premature rupture of membrane, fetal growth restriction, fetal hypoxia, premature birth, low birth weight and fetal death $[8,9]$. In low- and middle-income countries, $12 \%$ of low birth weight, $19 \%$ of preterm births, and $18 \%$ of perinatal mortality, are attributable to maternal anemia [10].

In China, a large quantity of deliveries takes place every year.. In 2016, for example, there were 18,466,561 live births 
[11]. Given the recently implemented two-child policy, reducing anemia and IDA during pregnancy has become a national priority for antenatal care. However, the available latest prevalence data was outdated that was from a survey $(n=3591)$ conducted nearly two decades ago [12].

In the last decade, China has undergone dramatic social and economic reforms, which exert profound impacts on pregnancy care, lifestyles, and maternal and child health. The lack of up-to-date information on the prevalence and risk factors of anemia and IDA has presented a major obstacle for developing evidence-based recommendations for managing pregnant women. To address such an important knowledge gap, we conducted a nationwide cross-sectional survey to investigate the current prevalence of anemia and IDA during pregnancy. Using a hybrid data collection process, the risk factors associated with the development of anemia and IDA for Chinese women were also ascertained in this study.

\section{Methods}

\section{Study design}

From 19 September to 20 November 2016, a nationwide cross-sectional survey was conducted at 24 hospitals from 16 provinces across China. The hospitals were selected according to the multi-stage sampling method (detailed below). Pregnant women, who attended these hospitals for antenatal care, were consecutively recruited into the study regardless of their gestational age.

The study was approved by the Research Ethics Committees of West China Second Hospital, Sichuan University (2016-009), Tongji Medical College of Huazhong University of Science and Technology (2016-S159), Beijing Friendship Hospital, Capital Medical University (2016-P2-058-01) and The First Affiliated Hospital of Zhengzhou University (2016-26). It was registered at ClinicalTrials.gov (ID number: NCT02887963). We have obtained informed consents from all participants. The Department of Obstetrics at West China Second University Hospital and the Chinese Evidence-based Medicine Center at West China Hospital, both from Sichuan University, took the primary responsibilities for study planning and design, project management, data collection, statistical analysis, and reporting.

\section{Sampling methods}

We used a multi-stage sampling method for selecting hospitals. According to the classification of the National Bureau of Statistics, the country consisted of six geographic regions (Supplementary Figure 1). First, from each region, we selected one teaching hospital as the regional coordinating center. Subsequently, three cities were randomly selected from that region. Then, from each selected city, we chose one tertiary hospital as a survey site based on our capacity and available resources. In total, 24 tertiary hospitals were included as survey sites (Supplementary Table 1).
The participating hospitals should meet the following criteria: 1) serum ferritin and hemoglobin were routinely tested at the local laboratory; 2) the laboratory facilities were examined by a quality control team comprising senior technicians, and 3) more than 400 pregnant women attending the hospital antenatal clinic during the survey period.

\section{Patient eligibility}

Our clinical investigators consecutively enrolled eligible pregnant women, regardless of their gestational age, who were visiting the selected hospitals for antenatal care. Eligibility criteria were: completed an antenatal visit between 19 September and 20 November 2016; agreed to take a blood test and complete the questionnaire survey, by signing an informed consent form. Those pregnant women who had been participating in another clinical study during the same period were excluded.

\section{Outcome measures}

The primary outcomes were the presence of anemia and IDA. According to the Chinese Guideline for Diagnosis and Treatment of IDA during Pregnancy [13], anemia was diagnosed by hemoglobin less than $110 \mathrm{~g} / \mathrm{l}$, and IDA was diagnosed by serum ferritin concentration less than $20 \mu \mathrm{g} / \mathrm{l}$ and hemoglobin less than $110 \mathrm{~g} / \mathrm{l}$.

All eligible participants were subjected to hemoglobin and serum ferritin examination during their antenatal visit. Blood samples were tested at the local laboratories using standard qualified methods.

We also documented previously clinician-diagnosed IDA during this pregnancy (i.e. first, second, or third trimester), which have been diagnosed by clinicians and recorded in medical charts.

\section{Data collection}

We used two structured questionnaires which had been pilot tested to collect information (Supplementary Table 2 and Table 3). The first questionnaire consisted of items regarding participant demographics, history of pregnancy and childbirth, gestational comorbidities, behavior and lifestyle, previously clinician-diagnosed IDA, and history of other gestational comorbidities. The second clinicianreported questionnaire was completed by manually reviewing electronic medical records (EMRs), including anthropometrics (e.g. height and weight), physical examinations, laboratory results (e.g. hemoglobin, serum ferritin, red blood cell count and leucocyte count), treatment of IDA, and maternal complications during pregnancy.

A centralized online electronic data capturing system was set up to collect, review, and store data. Participants either completed the questionnaire through WeChat or webpages, or a hardcopy version which was subsequently uploaded to the central system by trained investigators. 
Logic verification rules and reference ranges were implemented for data verification.

From each participating woman, we thoroughly collected the following information: demographic characteristics (maternal age, ethnicity, education level, local resident status, registered residential place (urban or rural) and annual family income), gestational characteristics (gestational weeks of pregnancy, multiple gestations, parity, use of assisted reproductive technology (ART), and nausea or vomiting during the first trimester (NVP)), anthropometrics (height, prepregnancy weight and current weight), behavior and habits (e.g. active or passive smoking, meat intake), nutrients supplementation (intake of folic acid, multivitamins and calcium supplements), and gestational co-morbidities (e.g. hematological diseases, HBV infection, hypertension, gynecological diseases, diabetes (Type I or Type II), thyroid and autoimmune diseases). Gestational co-morbidities were defined as those conditions diagnosed before conception by clinicians, or diagnoses at the first antenatal visit.

\section{Sample size estimation}

Prevalence data reported from a retrospective study was used for the sample size calculation [14]. Assuming a conservative prevalence estimate of maternal IDA being 3.96\% (i.e. $p \approx 4 \%$ ), the corresponding $\mathrm{d}$ (tolerance error) was taken to be 0.004 (one-tenth of $p$ ), with $Z_{\alpha / 2}=1.96$ and 2-tailed $\alpha=0.05$, the sample size required was $n=9220$.

Taking the available study resources and logistics into consideration, the investigators decided to increase the sample size to $n=12,000$. Each regional coordinating center was supposed to enroll at least 800 pregnant women, and the other 18 hospitals were expected to consecutively recruit over 400 participants at each site.

\section{Statistical analysis}

We summarized and compared baseline characteristics of pregnant women according to trimesters. Pearson's chisquare or Fisher's exact test were applied to categorical variables, and one-way ANOVA or Kruskal-Wallis test to continuous variables. Pre-pregnancy BMI was categorized into underweight (BMI $<18.5 \mathrm{~kg} / \mathrm{m}^{2}$ ), normal $\left(\mathrm{BMI} \geq 18.5 \mathrm{~kg} / \mathrm{m}^{2}\right.$ and $\left.<25 \mathrm{~kg} / \mathrm{m}^{2}\right)$, overweight and obese $\left(B M I \geq 25 \mathrm{~kg} / \mathrm{m}^{2}\right)$. We included women with complete data in our analyses.

We compared the observed hemoglobin and serum ferritin levels by study subgroup (singletons; multiple gestations) and by trimesters (first; second; third trimester), using means or medians as appropriate. Similarly, the frequencies and prevalence of anemia and IDA were compared with respect to trimesters and subgroups of interest.

To determine the risk factors associated with anemia and IDA, we considered all plausible factors from the literature, namely, demographic characteristics, gestational characteristics, anthropometry indicators, behavior and habits, nutrients supplementation, and gestational co- morbidities; details of these variables were given in the data collection section. Initially, associations with potential factors were explored by fitting univariate logistic regression models accounting for trimesters, with the significance level set at $p=0.1$. Next, we fitted mixed-effects logistic regression models for anemia and IDA separately. In view of the inherent correlation of observations due to the clustering effects of different hospitals, a mixed-effects logistic regression model with (24) random hospital effects was considered appropriate for our study setting.

\section{Sensitivity analysis}

In our study, we developed two sensitivity analyses. Firstly, to compare the prevalence of anemia during pregnancy with dissimilar races and settings, we used the different hemoglobin concentration thresholds for anemia diagnosis from other guidelines, involving UK guideline (i.e., anemia was defined as hemoglobin concentration $<110 \mathrm{~g} / \mathrm{l}$ in the first trimester, $<105 \mathrm{~g} / \mathrm{l}$ in second and third trimesters and $<100 \mathrm{~g} / \mathrm{l}$ postpartum and serum ferritin concentration $<30 \mu \mathrm{g} / \mathrm{l}$ ) [15] and WHO guideline (i.e., anemia was diagnosed as hemoglobin concentration $<110 \mathrm{~g} / \mathrm{l}$ in the first and third trimesters, and $<105 \mathrm{~g} / \mathrm{l}$ in the second trimester) [16], and reported the corresponding prevalence. Secondly, we conducted a mixed-effects logistic regression model with random hospital effects by removing pregnant women with a history of hematological diseases before pregnancy.

\section{Results}

Using a multi-stage sampling method, 24 hospitals from 21 cities of 16 provinces or municipal cities in China were enrolled in this study (Supplementary Figure 1). In total, 12,466 eligible pregnant women were invited to participate. After removing 63 refusals, 12,403 participants were included in the analysis (response rate: 99.5\%). The number of enrollments varied from 800 to 906 among the regional coordination centers, whereas recruitments were lower in the other hospitals (range: 400 to 443, Supplementary Table 1). The flow chart of the included sample of participants was given in Supplementary Figure 2.

\section{Baseline characteristics}

For our sample, 1018 women (8.2\%) were at the first trimester, $3487(28.1 \%)$ at the second, and 7898 (63.7\%) at the third. Table 1 presents the baseline characteristics of the participants by trimesters. The mean maternal age was 30 (SD 4) years, with the majority of participants being of Han ethnicity (93.1\%), living in urban area (51.5\%), attained higher education level (78.8\%), and had annual family income over 30 thousand yuan (90.6\%) (Table 1). It is evident that pregnant women at the three trimesters differs in a number of baseline characteristics $(p<0.05)$. 
Table 1 Characteristics of participants by trimesters

\begin{tabular}{|c|c|c|c|c|c|}
\hline Characteristics & Total $(n, \%)$ & First trimester $(n, \%)$ & $\begin{array}{l}\text { Second trimester } \\
(n, \%)\end{array}$ & $\begin{array}{l}\text { Third trimester } \\
(\mathrm{n}, \%)\end{array}$ & $P$ value \\
\hline Number & $12,403(100.0)$ & $1018(8.21)$ & $3487(28.11)$ & $7898(63.68)$ & $\underline{-}$ \\
\hline \multicolumn{6}{|l|}{ Regions } \\
\hline Southwest & $2105(16.97)$ & $214(21.02)$ & $693(19.87)$ & $1198(15.17)$ & \\
\hline East & $2008(16.19)$ & $123(12.08)$ & $484(13.88)$ & $1401(17.74)$ & \\
\hline Central-south & $2044(16.48)$ & $181(17.78)$ & $601(17.24)$ & $1262(15.98)$ & \\
\hline Northeast & $2179(17.57)$ & $38(3.73)$ & $469(13.45)$ & $1672(21.17)$ & \\
\hline Northwest & $2051(16.54)$ & $231(22.69)$ & 711 (20.39) & 1109 (14.04) & \\
\hline North & $2016(16.25)$ & $231(22.69)$ & $529(15.17)$ & $1256(15.90)$ & $<0.001^{1}$ \\
\hline \multicolumn{6}{|l|}{ Maternal age } \\
\hline$<35$ years & $10,595(85.42)$ & $893(87.72)$ & $2974(85.29)$ & $6728(85.19)$ & \\
\hline$\geq 35$ years & $1808(14.58)$ & $125(12.28)$ & $513(14.71)$ & $1170(14.81)$ & $0.09^{1}$ \\
\hline \multicolumn{6}{|l|}{ Ethnicity } \\
\hline Han & $11,542(93.06)$ & $959(94.20)$ & $3257(93.40)$ & $7326(92.76)$ & \\
\hline Others & $861(6.94)$ & $59(5.80)$ & $230(6.60)$ & $572(7.24)$ & $0.148^{1}$ \\
\hline \multicolumn{6}{|l|}{ Education level } \\
\hline$\geq 17$ years & $1376(11.09)$ & $120(11.79)$ & $372(10.67)$ & $884(11.19)$ & \\
\hline $13-16$ years & $8403(67.75)$ & 699 (68.66) & $2306(66.13)$ & $5398(68.35)$ & \\
\hline $10-12$ years & $1698(13.69)$ & $140(13.75)$ & $522(14.97)$ & $1036(13.12)$ & \\
\hline$\leq 9$ years & $926(7.47)$ & $59(5.80)$ & $287(8.23)$ & $580(7.34)$ & $0.02^{1}$ \\
\hline \multicolumn{6}{|l|}{ Local residents } \\
\hline No & $1394(11.24)$ & $120(11.79)$ & $463(13.28)$ & $811(10.27)$ & \\
\hline Yes & 11,009 (88.76) & $898(88.21)$ & 3024 (86.72) & 7087 (89.73) & $<0.001^{1}$ \\
\hline \multicolumn{6}{|c|}{ Registered residential place } \\
\hline Urban & $6367(51.33)$ & $516(50.69)$ & $1806(51.79)$ & $4045(51.22)$ & \\
\hline Rural & $6036(48.67)$ & $502(49.31)$ & $1681(48.21)$ & $3853(48.78)$ & $0.776^{1}$ \\
\hline \multicolumn{6}{|c|}{ Annual family income (thousand yuan) } \\
\hline$<30$ & $1167(9.41)$ & $72(7.07)$ & $320(9.18)$ & $775(9.81)$ & \\
\hline $30-79.9$ & $3427(27.63)$ & $263(25.83)$ & $994(28.51)$ & $2170(27.48)$ & \\
\hline $80-119.9$ & $3351(27.02)$ & $301(29.57)$ & $1010(28.96)$ & $2040(25.83)$ & \\
\hline $120-199.9$ & $2549(20.55)$ & $228(22.40)$ & $686(19.67)$ & $1635(20.70)$ & \\
\hline$\geq 200$ & $1909(15.39)$ & $154(15.13)$ & $477(13.68)$ & $1278(16.18)$ & $<0.001^{1}$ \\
\hline \multicolumn{6}{|c|}{ Multiple gestations } \\
\hline No & $12,121(97.84)$ & $996(96.70)$ & $3372(98.16)$ & $7753(97.73)$ & \\
\hline Yes & $282(2.16)$ & $22(3.30)$ & $115(1.84)$ & $145(2.27)$ & $<0.001^{1}$ \\
\hline \multicolumn{6}{|l|}{ Parity } \\
\hline Nulliparity & $8268(66.66)$ & $713(70.04)$ & $2290(65.67)$ & $5265(66.66)$ & \\
\hline Multiparity & $4135(33.34)$ & $305(29.96)$ & $1197(34.33)$ & $2633(33.34)$ & $0.034^{1}$ \\
\hline \multicolumn{6}{|l|}{ Use of ART } \\
\hline No & 11,921 (96.11) & 969 (95.19) & $3296(94.52)$ & 7656 (96.94) & \\
\hline Yes & $482(3.89)$ & $49(4.81)$ & $191(5.48)$ & $242(3.06)$ & $<0.001^{1}$ \\
\hline \multicolumn{6}{|l|}{ NVP } \\
\hline No & 2957 (23.84) & $167(16.40)$ & 715 (20.50) & $2075(26.27)$ & \\
\hline Slight & 7496 (60.44) & 671 (65.91) & $2196(62.98)$ & $4629(58.61)$ & \\
\hline Severe & 1950 (15.72) & 180 (17.68) & $576(16.52)$ & 1194 (15.12) & $<0.001^{1}$ \\
\hline
\end{tabular}


Table 1 Characteristics of participants by trimesters (Continued)

\begin{tabular}{|c|c|c|c|c|c|}
\hline Characteristics & Total $(n, \%)$ & First trimester $(n, \%)$ & $\begin{array}{l}\text { Second trimester } \\
(n, \%)\end{array}$ & $\begin{array}{l}\text { Third trimester } \\
(n, \%)\end{array}$ & $P$ value \\
\hline \multicolumn{6}{|l|}{ Pre-pregnancy BMI } \\
\hline Underweight & $2147(17.31)$ & $178(17.49)$ & $625(17.92)$ & $1344(17.02)$ & \\
\hline Normal weight & $8925(71.96)$ & $738(72.50)$ & $2467(70.75)$ & $5720(72.42)$ & \\
\hline Overweight and obese & $1331(10.73)$ & $102(10.02)$ & $395(11.33)$ & $834(10.56)$ & $0.405^{1}$ \\
\hline \multicolumn{6}{|l|}{ Active or passive smoking } \\
\hline No & $7710(57.37)$ & $584(62.46)$ & $2178(62.65)$ & $4948(62.16)$ & \\
\hline Yes & $4693(42.63)$ & $434(37.54)$ & $1309(37.35)$ & $2950(37.84)$ & $0.004^{1}$ \\
\hline Meat intake $\left(\mathrm{kg} /\right.$ week) ${ }^{\mathrm{a}}$ & $0.30(0.15-0.45)$ & $0.15(0.10-0.35)$ & $0.25(0.10-0.4)$ & $0.30(0.15-0.50)$ & $<0.001^{2}$ \\
\hline \multicolumn{6}{|l|}{ Intake of folic acid } \\
\hline No & $2077(16.75)$ & $181(17.78)$ & $530(15.20)$ & $1366(17.30)$ & \\
\hline Yes & $10,326(83.25)$ & $837(82.22)$ & $2957(84.80)$ & $6532(82.70)$ & $0.014^{1}$ \\
\hline \multicolumn{6}{|l|}{ Intake of multivitamins } \\
\hline No & $4305(34.71)$ & $517(50.79)$ & $1322(37.91)$ & $2466(31.22)$ & \\
\hline Yes & $8098(65.29)$ & $501(49.21)$ & $2165(62.09)$ & $5432(68.78)$ & $<0.001^{1}$ \\
\hline \multicolumn{6}{|l|}{ Intake of calcium } \\
\hline No & $4367(35.21)$ & $887(87.13)$ & $1664(47.72)$ & $1816(22.99)$ & \\
\hline Yes & $8036(64.79)$ & $131(12.87)$ & $1823(52.28)$ & $6082(77.01)$ & $<0.001^{1}$ \\
\hline \multicolumn{6}{|l|}{ Hematological diseases } \\
\hline No & 11,782 (94.99) & 987 (96.95) & $3365(96.50)$ & $7430(94.07)$ & \\
\hline Yes & $621(5.01)$ & $31(3.05)$ & $122(3.50)$ & $468(5.93)$ & $<0.001^{1}$ \\
\hline \multicolumn{6}{|l|}{ HBV infection } \\
\hline No & 12,097 (97.53) & 999 (98.13) & $3405(97.65)$ & $7693(97.40)$ & \\
\hline Yes & $306(2.47)$ & $19(1.87)$ & $82(2.35)$ & $205(2.60)$ & $0.323^{1}$ \\
\hline \multicolumn{6}{|l|}{ Hypertension } \\
\hline No & $12,363(99.68)$ & $1012(99.41)$ & $3473(99.60)$ & 7878 (99.75) & \\
\hline Yes & $40(0.32)$ & $6(0.59)$ & $14(0.40)$ & $20(0.25)$ & $0.128^{1}$ \\
\hline \multicolumn{6}{|l|}{ Gynecological diseases } \\
\hline No & 11,719 (94.49) & $933(91.65)$ & $3288(94.29)$ & $7498(94.94)$ & \\
\hline Yes & $684(5.51)$ & $85(8.35)$ & $199(5.71)$ & $400(5.06)$ & $<0.001^{1}$ \\
\hline \multicolumn{6}{|l|}{ Diabetes } \\
\hline No & $12,346(99.51)$ & $1013(99.51)$ & $3470(99.51)$ & $7863(99.56)$ & \\
\hline Yes & $57(0.49)$ & $5(0.49)$ & $17(0.49)$ & $35(0.44)$ & $0.938^{1}$ \\
\hline \multicolumn{6}{|l|}{ Thyroid diseases } \\
\hline No & $11,734(94.61)$ & 977 (95.97) & $3295(94.49)$ & $7462(94.48)$ & \\
\hline Yes & $669(5.39)$ & $41(4.03)$ & $192(5.51)$ & $436(5.52)$ & $0.131^{1}$ \\
\hline \multicolumn{6}{|l|}{ Autoimmune diseases } \\
\hline No & $12,356(99.62)$ & $1010(99.21)$ & $3461(99.25)$ & $7885(99.84)$ & \\
\hline Yes & $47(0.38)$ & $8(0.79)$ & $26(0.75)$ & $13(0.16)$ & $<0.001^{1}$ \\
\hline \multicolumn{6}{|l|}{ Digestive system diseases } \\
\hline No & 11,881 (95.79) & 949 (93.22) & 3331 (95.53) & 7601 (96.24) & \\
\hline Yes & $522(4.21)$ & 69 (6.78) & $156(4.47)$ & 297 (3.76) & $<0.001^{1}$ \\
\hline
\end{tabular}

${ }^{1}$ From Pearson's chi-square test

${ }^{2}$ From Kruskal-Wallis rank test

${ }^{a}$ Median (lower quartile-upper quartile)

$A R T$ assisted reproductive technology; NVP nausea or vomiting during pregnancy; HBV hepatitis B viral; BMI Body Mass Index 


\section{Hemoglobin and serum ferritin}

Table 2 summarizes the hemoglobin and serum ferritin levels by study subgroups and trimesters. It appears that both hemoglobin and serum ferritin tend to decrease with advancing trimesters, especially among women with multiple gestations (Table 2).

\section{Prevalence of anemia and IDA}

Table 3 presents the frequencies and prevalence of anemia and IDA by study subgroups and trimesters. A total of 2460 women were diagnosed with anemia; of whom 50 were identified at the first trimester, 577 at the second, and 1833 at the third. The overall prevalence of anemia was $19.8 \%(2460 / 12403)$, and $4.9 \%(50 / 1018)$ in the first trimester, $16.6 \%(577 / 3487)$ in the second trimester and $23.2 \%(1833 / 7898)$ in the third trimester. Besides, $13.9 \%(1720 / 12403)$ pregnant women were diagnosed with IDA, corresponding to $2.0 \%(20 / 1018)$ at the first trimester, $8.4 \%(293 / 3487)$ at the second, and $17.8 \%(1407 / 7898)$ at the third (Table 3).

Compared with singletons, women with multiple gestations experienced higher prevalence of anemia (28.7\% vs. $19.6 \%$ ) and IDA (20.6\% vs. $13.7 \%)$. Similarly, pregnant women with more than 35 years $(22.4 \%$ vs. $19.4 \%$ for anemia, $15.0 \%$ vs. $13.7 \%$ for IDA) and multiparity (22.7\% vs. $18.4 \%$ for anemia, $16.7 \%$ vs. $12.5 \%$ for IDA) presented higher prevalence of anemia and IDA than the counterparts (Table 3). The prevalence of anemia and IDA increased with trimesters, and peaked at the eighth month (24.0\% for anemia and $17.8 \%$ for IDA) (Fig. 1).

In addition, 5.2\% (53/1018), $17.0 \%$ (594/3487) and 39.9\% (3149/7898) pregnancies were previously cliniciandiagnosed with IDA at the three trimesters, respectively (Table 3). The prevalence also increased with the gestational month (Supplementary Figure 3).

Figure 2 shows that the prevalence of anemia and IDA varies substantially among the six regions of China. The southwest reported the lowest prevalence of anemia
(10.0\%) and IDA (15.4\%), whereas the east area had the highest prevalence in the second trimester (anemia $23.4 \%$ and IDA $13.3 \%$ ), and central-south had the highest prevalence in the third trimester (anemia $35.2 \%$ and IDA 28.8\%) (Fig. 2).

\section{Risk factors}

As shown in Table 4, the mixed-effects logistic regression analysis identified the following factors independently associated with a higher risk of anemia: late trimesters, maternal age 35 years or older, non-local residents, multiple gestations, multiparity, pre-pregnancy underweight, severe NVP during early pregnancy, preexisting IDA and other hematologic diseases. On the other hand, women who consume multivitamins and more meat were associated with a lower risk of anemia. (Table 4). Similar influencing factors in the same direction of associations were found for IDA, with the exception of maternal age (not significant; Table 4).

\section{Sensitivity analysis}

By using the different thresholds referring to anemia diagnosis, we found the overall prevalence of anemia by laboratory tests was $6.43 \%(796 / 12403)$ in UK guideline, corresponding to $4.91 \%(50 / 1018)$ in the first trimester, $7.66 \%(267 / 3487)$ in the second, and 6.09\% (481/7898) in the third. Combined with the thresholds of serum ferritin concentration less than $30 \mu \mathrm{g} / \mathrm{l}$, the prevalence of overall IDA was $5.14 \%$ (637/12403), corresponding to $2.26 \%(23 / 1018)$ in the first, $5.51 \%(192 / 3487)$ in the second and 5.34\% (422/7898) in the third trimester. Besides, compared with WHO's recommendation, we found the overall prevalence of anemia was $17.33 \%$ (2150/12403), corresponding to $4.91 \%(50 / 1018)$ in the first, $7.66 \%$ (267/3487) in the second, and $23.21 \%(1833 / 7898)$ in the third). In addition, the sensitivity analysis by removing pregnant women with hematological diseases diagnosed

Table 2 Average hemoglobin and serum ferritin levels of participants by trimesters

\begin{tabular}{|c|c|c|c|c|c|c|}
\hline Indicator & Sample & Total & First trimester & Second trimester & Third trimester & $P$ value \\
\hline \multirow[t]{3}{*}{$\begin{array}{l}\text { Hemoglobin: } \\
\text { mean (SD) }\end{array}$} & All pregnancies & $118.38(11.52)$ & $126.78(10.20)$ & $118.93(11.27)$ & 117.06 (11.32) & $\begin{array}{l}< \\
0.001^{1}\end{array}$ \\
\hline & Singletons & 118.43 (11.49) & $126.86(10.23)$ & 118.99 (11.25) & 117.10 (11.26) & $\begin{array}{l}< \\
0.001^{1}\end{array}$ \\
\hline & $\begin{array}{l}\text { Multiple } \\
\text { gestations }\end{array}$ & 116.47 (12.88) & $123.44(7.91)$ & 117.17 (11.73) & 114.86 (13.98) & $0.01^{1}$ \\
\hline \multirow{3}{*}{$\begin{array}{l}\text { Serum ferritin: } \\
\text { median (lower quartile-upper } \\
\text { quartile) }\end{array}$} & All pregnancies & $\begin{array}{l}20.60(11.79- \\
36.97)\end{array}$ & $54.39(34.5-94.01)$ & $\begin{array}{l}28.60(16.40- \\
50.50)\end{array}$ & $\begin{array}{l}16.70(10.20- \\
27.00)\end{array}$ & $\begin{array}{l}< \\
0.001^{2}\end{array}$ \\
\hline & Singletons & $20.57(11.7-36.80)$ & $\begin{array}{l}54.20(34.37- \\
93.35)\end{array}$ & $\begin{array}{l}28.74(16.40- \\
50.49)\end{array}$ & $16.63(10.20-26.9)$ & $\begin{array}{l}< \\
0.001^{2}\end{array}$ \\
\hline & $\begin{array}{l}\text { Multiple } \\
\text { gestations }\end{array}$ & 23.65 (13.9-44.44) & 75.7 (35.30-125.6) & $25.0(13.9-55.25)$ & $19.7(12.1-35.00)$ & $\begin{array}{l}< \\
0.001^{2}\end{array}$ \\
\hline
\end{tabular}

${ }^{1}$ From One-way ANOVA

${ }^{2}$ From Kruskal-Wallis rank test 
Table 3 Frequencies and prevalence of anemia and IDA

\begin{tabular}{|c|c|c|c|c|c|c|}
\hline Outcomes & Sample & Total $(n, \%)$ & $\begin{array}{l}\text { First trimester } \\
(\mathrm{n}, \%)\end{array}$ & $\begin{array}{l}\text { Second trimester } \\
(\mathrm{n}, \%)\end{array}$ & $\begin{array}{l}\text { Third trimester } \\
(\mathrm{n}, \%)\end{array}$ & $P$ value \\
\hline \multirow[t]{7}{*}{ Anemia } & All pregnancies & $2460(19.84)$ & $50(4.91)$ & $577(16.55)$ & $1833(23.21)$ & $<0.001$ \\
\hline & Singletons & $2379(19.63)$ & 49 (4.92) & $547(16.23)$ & $1783(23.00)$ & $<0.001$ \\
\hline & Multiple gestations & $81(28.72)$ & $1(4.55)$ & $30(26.09)$ & $50(34.48)$ & 0.011 \\
\hline & Maternal age $<35$ & $2055(19.40)$ & $42(4.70)$ & $477(16.04)$ & $1536(22.83)$ & $<0.001$ \\
\hline & Maternal age $\geq 35$ & $405(22.40)$ & $8(6.40)$ & $100(19.49)$ & $297(25.38)$ & $<0.001$ \\
\hline & Nullipara & $1520(18.39)$ & $31(4.36)$ & $372(16.25)$ & $1117(21.22)$ & $<0.001$ \\
\hline & Multipara & $940(22.73)$ & $19(6.23)$ & 205 (17.13) & 716 (27.19) & $<0.001$ \\
\hline \multirow[t]{7}{*}{ IDA } & All pregnancies & $1720(13.87)$ & $20(1.96)$ & $293(8.41)$ & $1407(17.82)$ & $<0.001$ \\
\hline & Singletons & $1662(13.72)$ & $19(1.91)$ & $276(8.19)$ & $1367(17.63)$ & $<0.001$ \\
\hline & Multiple gestations & $58(20.57)$ & $1(4.55)$ & $17(14.78)$ & $40(27.59)$ & 0.006 \\
\hline & Maternal age $<35$ & 1449 (13.68) & $17(1.90)$ & $247(8.31)$ & $1185(17.62)$ & $<0.001$ \\
\hline & Maternal age $\geq 35$ & $271(14.99)$ & $3(2.40)$ & $46(8.97)$ & $222(18.97)$ & $<0.001$ \\
\hline & Nullipara & $1031(12.47)$ & $10(1.40)$ & $174(7.40)$ & $847(16.09)$ & $<0.001$ \\
\hline & Multipara & $689(16.66)$ & $10(3.28)$ & $119(9.94)$ & $560(21.27)$ & $<0.001$ \\
\hline \multirow[t]{7}{*}{ Previously clinician-diagnosed IDA } & All pregnancies & $3796(30.61)$ & $53(5.21)$ & $594(17.03)$ & $3149(39.87)$ & $<0.001$ \\
\hline & Singletons & $3699(30.52)$ & $52(5.22)$ & $564(16.73)$ & $3083(39.77)$ & $<0.001$ \\
\hline & Multiple gestations & $97(34.52)$ & $1(4.55)$ & $30(26.09)$ & $66(45.52)$ & $<0.001$ \\
\hline & Maternal age $<35$ & $3226(30.45)$ & $48(5.38)$ & $510(17.15)$ & $2668(39.66)$ & $<0.001$ \\
\hline & Maternal age $\geq 35$ & $570(31.53)$ & $5(4.00)$ & $84(16.37)$ & $481(41.11)$ & $<0.001$ \\
\hline & Nullipara & $2415(29.21)$ & $37(5.19)$ & $360(15.72)$ & $2081(38.33)$ & $<0.001$ \\
\hline & Multipara & $1381(33.40)$ & $16(5.25)$ & $234(19.55)$ & $1131(42.95)$ & $<0.001$ \\
\hline
\end{tabular}

'From Pearson's chi-square test

IDA iron deficiency anemia

before pregnancy confirmed the similar results (not presented for brevity).

\section{Discussion}

\section{Prevalence of anemia and IDA}

Using a large representative sample of pregnant women, we found that $19.8 \%$ of Chinese women had anemia and $13.9 \%$ had IDA during pregnancy, and the conditions became more prevalent over the progression of gestation. The prevalence of anemia and IDA also varied substantially across geographic regions of China, from the lowest in the southwest to the highest in the central-south.

In China, anemia in pregnancy was diagnosed as hemoglobin concentrations $<110 \mathrm{~g} / \mathrm{l}$ throughout three trimesters. When we used the newest UK's guideline for assessing anemia, we found the prevalence of anemia was consistent in the first trimester, but much lower than those diagnosed by Chinese guideline for overall anemia (i.e., $6.43 \%$ vs. $19.84 \%$ ). On the other hand, WHO's recommendation defined the different threshold in the second trimester only, thus having slightly lower overall prevalence of anemia (i.e., $17.33 \%$ vs. $19.84 \%$ ). Due to physiological expansion of plasma volume in pregnancy, especially in the second and third trimesters [17], setting different thresholds for defining anemia diagnosis in pregnancy seems to be plausible. However, the current hemoglobin concentrations thresholds during pregnancy derived from non-pregnant populations, and there was limited evidence to clarify the association between those thresholds and subsequently clinical outcomes [18]. Additionally, it was reported that racial disparities were not negligible in maternal hemoglobin concentrations and pregnancy outcomes [19]. Therefore, we adopted the Chinese guideline in our main analysis, being consistent with Chinese practice, and supplemented a sensitivity analysis to compare the prevalence of anemia according to other thresholds.

On the other hand, IDA was defined as anemia plus serum ferritin $<20 \mu \mathrm{g} / \mathrm{l}$ in our study, whereas different thresholds were used in other countries [20, 21], for example, < $10 \mu \mathrm{g} / \mathrm{l}$ USA [22]; < $15 \mu \mathrm{g} / \mathrm{l}$ Korea [23]; <12$15 \mu \mathrm{g} / \mathrm{l}$ Europe [5]; and $<30 \mu \mathrm{g} / \mathrm{l}$ Switzerland [24] and UK [15]. Accordingly, the direct comparison of prevalence of IDA among countries were actually difficult. In sensitivity analysis, we defined IDA according to UK's guideline, thus presenting the lower prevalence of overall IDA $(5.14 \%$ vs. 13.87\%). In addition, our study found rapid decline in hemoglobin and serum ferritin levels with advancing trimesters, consistent with the literatures $[5,25,26]$. The 


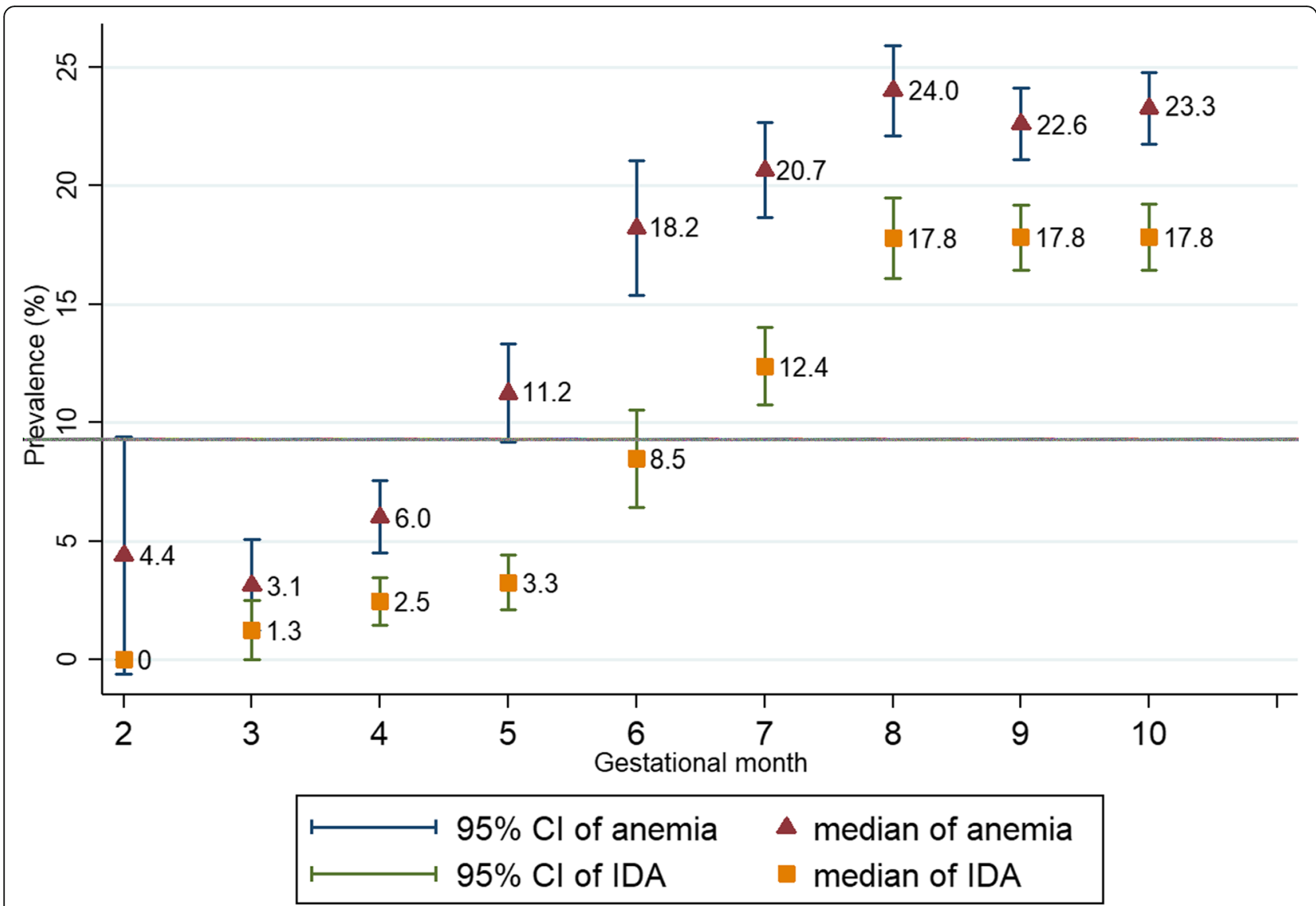

Fig. 1 Prevalence of anemia and IDA at each gestational month

prevalence of anemia and IDA peaked at the eighth gestational month according to diagnosis in Chinese guideline.

A recent systematic analysis (2011) reported that the global prevalence of anemia among pregnant women was $38 \%$ [3]. In developed countries, the prevalence was lower (for example, anemia: $12.8 \%$ in Canada [27], $15.8 \%$ in France [28] and $8.8 \%$ in U. S [29]; IDA: $16 \%$ in Belgian, and 3\% in Switzerland), but the prevalence remained high in developing nations (for example, anemia: $53.4 \%$ in Congo [7]); IDA: 51.3\% in Egypt [6] and 44.5\% in Saudi Arabia [30]). Besides, the prevalence of anemia in pregnant population varied across Asia-pacific regions, such as 25.7\% in Korea [5], 20-25\% in Australia [31-33] and 50\% in India [34]. According to the estimates by WHO, the lowest prevalence of anemia during pregnancy occurred in Western Pacific region (24.3\%) and the highest prevalence in South-East Asia region (48.7\%) [35].

Our findings were similar to those from developed countries. This suggests that pregnancy care for the Chinese population has improved over the past decades. However, due to different diagnostic criteria adopted by studies, one should interpret the findings with caution [36-38].

In addition, we found the prevalence of anemia and IDA varied substantially among the six regions of China.
Except for the impact of socioeconomic status, the difference of dietary habit and region-epidemic of thalassemia among Chinese provinces and races, might play important role in diverse distribution of anemia and IDA. These factors were also used to illustrate the high prevalence of anemia in South-East Asia, such as high epidemic of thalassemia in Thailand, Laos and Philippines, and low-iron dietary intake and sickle-cell anemia in India [39-41].

\section{Risk factors for anemia and IDA}

Several risk factors were identified for anemia and IDA. The magnitudes of association for pertinent factors such as advanced trimesters, multiple gestations, IDA prior to pregnancy, and hematological diseases other than IDA, were relatively strong (adjusted odds ratio over 2). The results were consistent with studies on non-Chinese populations [42-45], and have important implications for managing pregnant women with these characteristics who may be at elevated risk. The sensitivity analysis by excluding those with previously diagnosed IDA or anemia have further confirmed the apparent adverse effect of these factors on the development of anemia or IDA. 

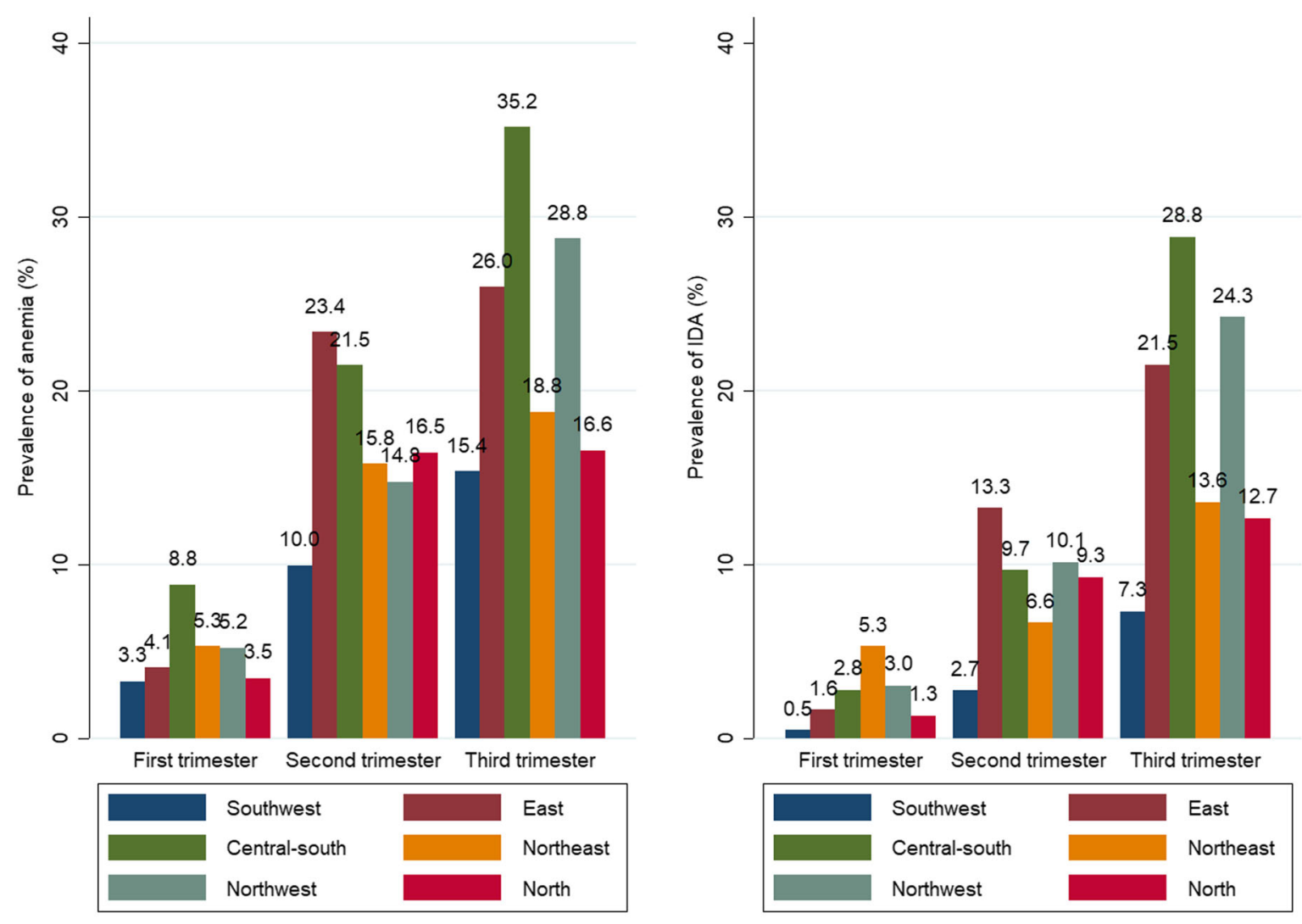

Fig. 2 Prevalence of anemia and IDA among the six regions of China

In addition, several other factors were found to be moderately associated with the prevalence of anemia and IDA (adjusted odds ratios between 1.2 and 1.3), including nonlocal residents, multiparity, severe NVP, and prepregnancy underweight, similar to findings reported in the literature [23, 45-50]. These factors should also be taken into account when planning for interventions to improve the anemia and IDA status of Chinese pregnant women.

\section{Supplementation and screening}

Multivitamins supplement and meat intake were associated with lower risk of anemia and IDA. Multivitamins products, which contain vitamins and minerals including iron $[51,52]$, are commonly consumed in China. The finding concerning meat intake was consistent with previous studies in Ethiopia [24, 53, 54], in which IDA was found more prevalent in regions with iron-poor diets such as central south, northwest, and east China [23, 55].

Prophylactic iron supplementation for pregnant women is a controversial issue [36-38]. Although the current Chinese guideline does not address such supplementation [13], routine iron supplementation during pregnancy have been suggested in 24 hospitals. Accordingly, the prevalence of anemia and IDA were substantially lower than national investigation conducted before 20 years in China [12]. Our findings underlined that iron supplements and dietary improvement may be warranted for susceptible and high-risk population subgroups, such as pregnant women with multiple gestations, more than 35 years and multiparity. It has been recommended that all pregnant women should be screened for anemia during pregnancy $[22,56,57]$. However, the timing of screening remains uncertain. At present, the Chinese guideline recommends screening of hemoglobin at the first antenatal visit, with repeated screening every $8-10$ weeks [13]. Our result supports such screening, especially at the advanced trimesters. In addition, although serum ferritin test may be impacted by inflammatory or infectious conditions, a routine marker for diagnosis of iron deficiency was advisable during pregnancy in light of reliably automated operation and obvious cost-effectiveness compared with other markers (e.g., transferrin receptor) [20, 58]. A concurrent test for inflammatory markers (e.g., C-reactive protein) is considered to exclude other reactive causes [20,59]. 
Table 4 Risk factors for anemia and IDA

\begin{tabular}{|c|c|c|}
\hline Factors & Anemia: adjusted OR $(95 \% \mathrm{Cl})^{\mathrm{a}}$ & IDA: adjusted OR $(95 \% \mathrm{Cl})^{\mathrm{b}}$ \\
\hline \multicolumn{3}{|l|}{ Trimester } \\
\hline Second vs. First & $4.11(3.02-5.57)$ & $4.99(3.13-7.95)$ \\
\hline Third vs. First & $6.10(4.52-8.22)$ & $11.72(7.43-18.49)$ \\
\hline Maternal age ( $\geq 35$ vs. $<35$ years) & $1.22(1.06-1.40)$ & - \\
\hline Local residents (No vs. Yes) & $1.18(1.02-1.36)$ & $1.20(1.02-1.42)$ \\
\hline Multiple gestations (Yes vs. No) & $1.54(1.13-2.09)$ & $1.90(1.38-2.62)$ \\
\hline Parity (Multiparity vs. Nulliparity) & $1.18(1.06-1.31)$ & $1.28(1.14-1.44)$ \\
\hline \multicolumn{3}{|l|}{ Pre-pregnancy BMI } \\
\hline Underweight vs. Normal weight & $1.32(1.17-1.49)$ & $1.30(1.13-1.49)$ \\
\hline Overweight and obese vs. Normal weight & $0.67(0.57-0.80)$ & $0.62(0.51-0.77)$ \\
\hline \multicolumn{3}{|l|}{ NVP } \\
\hline Slight vs. No & $1.05(0.93-1.18)$ & $1.06(0.93-1.22)$ \\
\hline Severe vs. No & $1.21(1.04-1.41)$ & $1.21(1.02-1.44)$ \\
\hline Intake of multivitamins (Yes vs. No) & $0.58(0.52-0.65)$ & $0.49(0.43-0.55)$ \\
\hline IDA before pregnancy (Yes vs. No) & $2.92(2.41-3.54)$ & $2.81(2.28-3.48)$ \\
\hline Other hematologic diseases (Yes vs. No) & $8.25(5.19-13.13)$ & $2.00(1.25-3.20)$ \\
\hline Meat intake (kg/week) & $0.81(0.68-0.96)$ & $0.80(0.65-0.98)$ \\
\hline
\end{tabular}

\section{Strengths and limitations}

Our study has several advantages. In this nationwide survey, a multi-stage sampling method was applied to select the data collection sites. To our knowledge, this was the largest study ever undertaken in China to investigate the prevalence and risk factors of anemia and IDA during pregnancy. Moreover, rigorous methods were adopted for data collection, ensuring high quality information obtained across the country with minimal missing values. The large sample size has enabled the estimation of overall prevalence of anemia and IDA, as well as facilitated the comparison of rates among regions, population subgroups, and across trimesters.

Several limitations should be taken into consideration. Firstly, due to logistics problem, hemoglobin and serum ferritin were tested at local laboratories, where the diagnostic accuracy may vary slightly. To circumvent the problem, each participating tertiary hospital was required to be equipped with qualified facilities and professional technicians, and a laboratory quality control team was set up to minimize measurement errors, thus ensuring the reliability and comparability of results. Secondly, random sampling for hospital selection was not feasible due to the vastness of regions and economic constraints. We chose this approach to optimize the balance between available resource and the representativeness of our study locations. Thirdly, all the participating data collection sites were tertiary hospitals with laboratory facilities, the generalizability of findings may be slightly compromised without the inclusion of smaller hospitals and community clinics.

\section{Conclusion}

The prevalence of anemia and IDA during pregnancy are similar to those from developed countries and vary across regions in China. However, due to different diagnostic criteria adopted, one should compare the findings with other studies with caution. Non-local residents, multiple gestations, multiparity, pre-pregnancy underweight, severe NVP, hematologic diseases before pregnancy and other hematologic diseases rather than IDA were associated with higher risks of anemia and IDA. Our study highlights the need for developing and implementing a rigorous management plan to control pregnancy anemia and IDA in China.

\section{Supplementary Information}

The online version contains supplementary material available at https://doi. org/10.1186/s12884-020-03359-z.

Additional file 1 Figure $\mathbf{S 1}$ The distribution of six regions and selected cities among China. Figure $\mathbf{S 2}$ The flow chart of included population. Figure S3 Median and 95\% confidence intervals for prevalence of previously diagnosed IDA categorized by gestational month. Table S1 List of 24 selected hospitals in our study and the number of included pregnant women. Table S2 Survey on iron deficiency among pregnant women in China (Pregnant Women Investigation). Table S3 Survey on iron deficiency among pregnant women in China (Doctor Investigation) 


\section{Abbreviations}

IDA: Iron deficiency anemia; EMR: Electronic medical records; ART: Assisted reproductive technology; NVP: Nausea or vomiting during pregnancy; HBV: Hepatitis B viral; BMI: Body mass index; aOR: Adjusted Odds Ratio; $\mathrm{Cl}$ : Confidence interval

\section{Acknowledgments}

Steering Committee: Xing-hui Liu, Xin Sun, Jing He, Li Zou, Caixia Liu, Yinli Cao, Ling Fan, Xu Chen, Guo-lin He and Jing Tan.

Methods Coordination Center: Xin Sun, Jing Tan, Xing-hui Liu, Ya-na Qi and Kang Zou.

List of investigators from 24 hospitals: West China Second Hospital, Sichuan University (Xinghui Liu, Guolin He, Lingling Peng \& Hongmei Yang); Sichuan Provincial Hospital for Women and Children (Yan Gao), Panzhihua Central Hospital (Xiuli Liu); Chongqing Health Center for Women and Children (Wei Zhou), Women's Hospital School of Medicine, Zhejiang University (Jing He \& Lu Chen); Xiamen Medical Center for Women and Children (Xueqin Zhang); Wenzhou People's Hospital (Hongping Zhang); Suzhou Second People's Hospital/ Suzhou Municipal Hospital (Yun Wang \& Xiufen Xu); Union Hospital, Tongji Medical College, Huazhong University of Science and Technology (Li Zou); Maternal and Child Health Hospital of Hubei Province (Mei Xiao \& Min Peng); The First Affiliated Hospital of Zhengzhou University (Xianlan Zhao \& Yan Zhou); The First Affiliated Hospital of Guangxi Medical University (Hui Tang); Shengjing Hospital of China Medical University (Caixia Liu \& Chong Qiao); Maternal and Child Health Hospital of Dalian (Fang Zhan); Changchun Obstetrics-Gynecology Hospital (Chunxia Yin); The Fourth Affiliated Hospital of Harbin Medical University (Yan Cai); Shanxi Provincial Hospital for Women and Children/ Northwest Women's and Children's Hospital (Yinli Cao); The Second Affiliated Hospital of Shanxi University of Traditional Chinese Medicine (Ningxia Yuan); Yan'an University Affiliated Hospita (Hongmei Li); Maternal and Child Health Hospital of Urumchi, Xinjiang (Guifeng Ding \& Yan Peng); Beijing Obstetrics and Gynecology Hospital, Capital Medical University (Ling Fan \& Jieyan Li); Tianjin Central Hospital of Gynecology Obstetrics (Xu Chen); The Fourth Hospital of Shijiazhuang/ Shijiazhuang Obstetrics and Gynecology Hospital (Guohua Zhang); and Beijing Friendship Hospital, Capital Medical University (Jiewen Zhang), for their efforts for participants enrollment and data collection.

\section{Authors' contributions}

Study conceptualization and design: XHL, XS, JT. Study management and oversight: XHL, JT, GLH, YNQ, HMY. Data analysis: JT, WW. Draft of the manuscript: JT. Critical revision: AL, XS, YNQ, YQX, CRL, KZ. All authors read and approved the final version of the manuscript.

\section{Funding}

This study was funded by The National Key Research and Development Program of Reproductive Health \& Major Birth Defects Control and Prevention (No.2016YFC1000406), The National Natural Science Foundation of China (No. 71704122, 81590955), The National Science and Technology Major Project (No. 2018ZX10302206), The National Key Development Plan for Precision Medicine Research (No. 2017YFC0910004), and "Thousand Youth Talents Plan" of China (No. D1024002). They had no role in the design of the study, the collection, analysis, and interpretation of data and in writing the manuscript.

\section{Availability of data and materials}

The datasets analyzed during the current study are not currently available due to patient confidentiality reasons as well as the use of the same data for developing other manuscripts.

\section{Ethics approval and consent to participate}

The study was approved by the Research Ethics Committee of West China Second Hospital, Sichuan University (2016-009), and each participant should sign an informed consent form.

\section{Consent for publication}

All participants have consented to the publication of study results.

\section{Competing interests}

The authors declare that they have no competing interests.

\section{Author details}

'Chinese Evidence-based Medicine Center, West China Hospital, Sichuan University, No.37 Guo Xue Xiang, Chengdu, China. ${ }^{2}$ Department of Obstetrics and Gynecology, and Key Laboratory of Birth Defects and Related Diseases of Women and Children (Sichuan University), Ministry of Education; West China Second University Hospital, Sichuan University, No.37 Guo Xue Xiang, Chengdu, China. ${ }^{3}$ School of Public Health, Curtin University, Perth, WA, Australia.

Received: 14 February 2020 Accepted: 27 October 2020

Published online: 07 November 2020

\section{References}

1. Goonewardene M, Shehata M, Hamad A. Anaemia in pregnancy. Best practice \& research Clinical obstetrics \& gynaecology. 2012;26:3-24. https:// doi.org/10.1016/j.bpobgyn.2011.10.010.

2. World Health Organization, In de Benoist B. In: Mclean E, Egli I, Cogswell M, editors. Worldwide prevalence of anaemia 1993-2005. WHO Global Database on Anaemia. Geneva: World Health Organization; 2008. (NLM: WH 155).

3. Stevens GA, Finucane MM, De-Regil LM, Paciorek CJ, Flaxman SR, Branca F, et al. Global, regional, and national trends in haemoglobin concentration and prevalence of total and severe anaemia in children and pregnant and non-pregnant women for 1995-2011: a systematic analysis of populationrepresentative data. Lancet Glob Health. 2013;1:e16-25. https://doi.org/10. 1016/s2214-109x(13)70001-9.

4. International Nutritional Anemia Consultative Group (INACG) WHOW, United Nations Childrens Fund (UNICEF). Guidelines for the Use of Iron Supplements to Prevent and Treat Iron Defciency Anemia. Stoltzfus RJ, ML D. Washington DC: ILSI Press; 1998.

5. CLT NM, Merkel J, Brannon PM. Iron status in pregnant women and women of reproductive age in Europe. Am J Clin Nutrition. 2017;106(Suppl). https:// doi.org/10.3945/ajen.

6. Rezk M, Marawan H, Dawood R, Masood A, Abo-Elnasr M. Prevalence and risk factors of iron-deficiency anaemia among pregnant women in rural districts of Menoufia governorate, Egypt. J Obstetrics Gynaecology. 2015;35: 663-6. https://doi.org/10.3109/01443615.2014.991289.

7. Tandu-Umba B, Mbangama AM. Association of maternal anemia with other risk factors in occurrence of Great obstetrical syndromes at university clinics, Kinshasa, DR Congo. BMC Pregnancy Childbirth. 2015;15:183. https://doi.org/ 10.1186/s12884-015-0623-z.

8. Haider BA, Olofin I, Wang M, Spiegelman D, Ezzati M, Fawzi WW, et al. Anaemia, prenatal iron use, and risk of adverse pregnancy outcomes: systematic review and meta-analysis. Bmj. 2013;346:f3443. https://doi.org/10.1136/bmj.f3443.

9. Iqbal S, Ekmekcioglu C. Maternal and neonatal outcomes related to iron supplementation or iron status: a summary of meta-analyses. J Maternal-Fetal Neonatal Med. 2017:1-13. https://doi.org/10.1080/14767058.2017.1406915.

10. Rahman MM, Abe SK, Rahman MS, Kanda M, Narita S, Bilano V, et al. Maternal anemia and risk of adverse birth and health outcomes in low- and middle-income countries: systematic review and meta-analysis. Am J Clin Nutr. 2016;103:495-504. https://doi.org/10.3945/ajcn.115.107896.

11. National Health and Family Planning Commission. China Health and Family Planning Statistical Yearbook: http://www.yearbookchina.com/navibooklistN2017120225-1.html 2017.

12. Chinese collaboration group for epidemiological investigation of iron deficiency in children, pregnant women and women of childbearing age. Prevalence of iron deficiency in pregnant women and women of childbearing age in China. Chin J Hematology. 2004;25:653-7.

13. Society of Perinatal Medicine, Chinese Medical Association. Guideline for diagnosis and treatment of iron deficiency and iron deficiency anemia in pregnancy. Chin J Perinat Med. 2014;17:451-4.

14. Tan J, Liu XH, Yu C, Chen M, Chen XF, Sun X, et al. Effects of medical comorbidities on severe maternal morbidities in China: a multicenter clinic register study. Acta Obstet Gynecol Scand. 2015;94:861-8. https://doi.org/10. 1111/aogs.12657.

15. Pavord S, Daru J, Prasannan N, Robinson S, Stanworth S, Girling J, et al. UK guidelines on the management of iron deficiency in pregnancy. $\mathrm{Br} J$ Haematol. 2020;188:819-30. https://doi.org/10.1111/bjh.16221.

16. WHO. WHO recommendations on antenatal care for a positive pregnancy experience https://apps.who.int/iris/bitstream/handle/10665/250796/ 9789241549912-eng.pdf;isessionid=E983BD96368F7B3C08E93F02A75 FADFD? sequence $=12016$. 
17. Costantine MM. Physiologic and pharmacokinetic changes in pregnancy Front Pharmacol. 2014;5:65. https://doi.org/10.3389/fphar.2014.00065.

18. Pasricha SR, Colman K, Centeno-Tablante E, Garcia-Casal MN, Peña-Rosas JP. Revisiting WHO haemoglobin thresholds to define anaemia in clinical medicine and public health. Lancet Haematology. 2018;5:e60-e2. https:// doi.org/10.1016/s2352-3026(18)30004-8.

19. Mohamed MA, Ahmad T, Macri C, Aly H. Racial disparities in maternal hemoglobin concentrations and pregnancy outcomes. J Perinat Med. 2012; 40:141-9. https://doi.org/10.1515/jpm.2011.137.

20. Khalafallah AA, Dennis AE. Iron deficiency anaemia in pregnancy and postpartum: pathophysiology and effect of oral versus intravenous iron therapy. J Pregnancy. 2012;630519. https://doi.org/10.1155/2012/630519.

21. Daru J, Allotey J, Pena-Rosas JP, Khan KS. Serum ferritin thresholds for the diagnosis of iron deficiency in pregnancy: a systematic review. Transfus Med. 2017:27:167-74. https://doi.org/10.1111/tme.12408.

22. Committee ACOG. On practice bulletins. ACOG practice bulletins: clinical management guidelines for obstetrician-gynecologists no. 95: anemia in pregnancy. Obstet Gynecol. 2008;112:201-7. https://doi.org/10.1097/AOG. Ob013e3181809c0d.

23. Lee JO, Lee JH, Ahn S, Kim JW, Chang H, Kim YJ, et al. Prevalence and risk factors for iron deficiency anemia in the korean population: results of the fifth KoreaNational health and nutrition examination survey. J Korean Med Sci. 2014;29:224-9. https://doi.org/10.3346/jkms.2014.29.2.224.

24. Abay A, Yalew HW, Tariku A, Gebeye E. Determinants of prenatal anemia in Ethiopia. Archives of public health $=$ Archives belges de sante publique. 2017;75: 51. https://doi.org/10.1186/s13690-017-0215-7.

25. Bah A, Pasricha SR, Jallow MW, Sise EA, Wegmuller R, Armitage AE, et al. Serum Hepcidin concentrations decline during pregnancy and may identify Iron deficiency: analysis of a longitudinal pregnancy cohort in the Gambia. J Nutr. 2017;147:1131-7. https://doi.org/10.3945/jn.116.245373.

26. van Santen S, Kroot JJ, Zijderveld G, Wiegerinck ET, Spaanderman ME, Swinkels DW. The iron regulatory hormone hepcidin is decreased in pregnancy: a prospective longitudinal study. Clin Chem Lab Med. 2013;51: 1395-401. https://doi.org/10.1515/cclm-2012-0576.

27. Smith C, Teng F, Branch E, Chu S, Joseph KS. Maternal and perinatal morbidity and mortality associated with Anemia in pregnancy. Obstet Gynecol. 2019;134:1234-44. https://doi.org/10.1097/AOG.0000000000003557.

28. Thierry Harvey AZ, Auges M, Clavel T. Assessment of iron deficiency and anemia in pregnant women: an observational French study. Womens Health; 2015.

29. Le CH. The prevalence of Anemia and moderate-severe Anemia in the US population (NHANES 2003-2012). PLoS One. 2016;11:e0166635. https://doi. org/10.1371/journal.pone.0166635.

30. Baradwan S, Alyousef A, Turkistani A. Associations between iron deficiency anemia and clinical features among pregnant women: a prospective cohort study. J Blood Med. 2018;9:163-9. https://doi.org/10.2147/JBM.S175267.

31. Frayne J, Pinchon D. Anaemia in pregnancy. Aust J General Practitioners 2019:48:125-9

32. Khalafallah A, Dennis A, Bates J, Bates G, Robertson IK, Smith L, et al. A prospective randomized, controlled trial of intravenous versus oral iron for moderate iron deficiency anaemia of pregnancy. J Intern Med. 2010;268: 286-95. https://doi.org/10.1111/j.1365-2796.2010.02251.x.

33. Khalafallah AA, Hyppa A, Chuang A, Hanna F, Wilson E, Kwok C, et al. A prospective randomised controlled trial of a single intravenous infusion of ferric Carboxymaltose vs single intravenous Iron Polymaltose or daily Oral ferrous Sulphate in the treatment of Iron deficiency Anaemia in pregnancy. Semin Hematol. 2018:55:223-34. https://doi.org/10.1053/.seminhematol.2018.04.006

34. Kalaivani K, Ramachandran P. Time trends in prevalence of anaemia in pregnancy. Indian J Med Res. 2018;147:268-77. https://doi.org/10.4103/ijmr. IJMR173016.

35. WHO. The global prevalence of anaemia in 2011. Geneva: World Health Organization 2015 https://appswhoint/iris/bitstream/handle/10665/177094/ 9789241564960_engpdf?sequence=h81.

36. Pavord S, Myers B, Robinson S, Allard S, Strong J, Oppenheimer C. UK guidelines on the management of iron deficiency in pregnancy. $\mathrm{Br} J$ Haematol. 2012;156:588-600.

37. WHO. Guideline: Daily iron and folic acid supplementation in pregnant women. 2012.

38. Peyrin-Biroulet LWN, Cacoub P. Guidelines on the diagnosis and treatment of iron deficiency across indications: a systematic review. Am J Clin Nutr. 2015;102:1585-94.
39. Fucharoen S, Winichagoon P. Haemoglobinopathies in Southeast Asia. Indian J Med Res. 2011.

40. McMahon LP. Iron deficiency in pregnancy. Obstet Med. 2010;3:17-24. https://doi.org/10.1258/om.2010.100004

41. WHO. Sickle-cell anaemia Report by the Secretariat. FIFTY-NINTH WORLD HEALTH ASSEMBLY (Provisional agenda item 114) 2006 https://appswhoint/ gb/archive/pdf_files/WHA59/A59_9-enpdf..

42. Sukrat B, Suwathanapisate $P$, Siritawee S, Poungthong T, Phupongpankul K. The prevalence of iron deficiency anemia in pregnant women in Nakhonsawan, Thailand. Journal of the medical Association of Thailand $=$. Chotmaihet thangphaet. 2010;93:765-70.

43. Gebreweld A, Tsegaye A. Prevalence and Factors Associated with Anemia among Pregnant Women Attending Antenatal Clinic at St. Paul's Hospital Millennium Medical College, Addis Ababa, Ethiopia. Adv Hematology. 2018; 3942301. https://doi.org/10.1155/2018/3942301.

44. Ru Y, Pressman EK, Cooper EM, Guillet R, Katzman PJ, Kent TR, et al. Iron deficiency and anemia are prevalent in women with multiple gestations. Am J Clin Nutr. 2016;104:1052-60. https://doi.org/10.1007/s00125-016-4149-3.

45. Karaoglu L, Pehlivan E, Egri M, Deprem C, Gunes G, Genc MF, et al. The prevalence of nutritional anemia in pregnancy in an east Anatolian province, Turkey. BMC Public Health. 2010;10:329. https://doi.org/10.1186/1471-2458-10-329.

46. Mocking M, Savitri Al, Uiterwaal C, Amelia D, Antwi E, Baharuddin M, et al. Does body mass index early in pregnancy influence the risk of maternal anaemia? An observational study in Indonesian and Ghanaian women. BMC Public Health. 2018;18:873. https://doi.org/10.1186/s12889-018-5704-2.

47. Tan J, Qi YN, He GL, Yang HM, Zhang GT, Zou K, et al. Association between maternal weight indicators and Iron deficiency Anemia during pregnancy: a cohort study. Chin Med J. 2018;131:2566-74. https://doi.org/10.4103/03666999.244109.

48. Baig-Ansari N, Badruddin SH, Karmaliani R, Harris H, Jehan I, Pasha O, et al. Anemia prevalence and risk factors in pregnant women in an urban area of Pakistan. Food Nutr Bull. 2008;29:132-9. https://doi.org/10.1177/156482650802900207.

49. Bencaiova G, Burkhardt T, Breymann C. Anemia--prevalence and risk factors in pregnancy. Eur J Internal Med. 2012;23:529-33. https://doi.org/10.1016/j. ejim.2012.04.008.

50. Fiaschi L, Nelson-Piercy C, Gibson J, Szatkowski L, Tata LJ. Adverse maternal and birth outcomes in women admitted to Hospital for Hyperemesis Gravidarum: a population-based cohort study. Paediatr Perinat Epidemiol. 2018;32:40-51. https://doi.org/10.1111/ppe.12416.

51. Ning $M$, Chen $Y$, Zheng Q, Jia J, Bai B. Insignificant interference of Elevit in pregnant women serum samples with HBsAg immunoassay on Sysmex. J Clin Lab Anal. 2018: e22725. https://doi.org/10.1002/jcla.22725.

52. Diemert A, Lezius S, Pagenkemper M, Hansen G, Drozdowska A, Hecher K, et al. Maternal nutrition, inadequate gestational weight gain and birth weight: results from a prospective birth cohort. BMC pregnancy and childbirth. 2016;16:224. https://doi.org/10.1186/s12884-016-1012-y.

53. Weldekidan F, Kote M, Girma M, Boti N, Gultie T. Determinants of Anemia among pregnant women attending antenatal Clinic in Public Health Facilities at Durame town: unmatched case control study. Anemia. 2018; 8938307. https://doi.org/10.1155/2018/8938307.

54. Obse N, Mossie A, Gobena T. Magnitude of anemia and associated risk factors among pregnant women attending antenatal care in Shalla Woreda, west Arsi zone, Oromia region, Ethiopia. Ethiop J Health Sci. 2013;23:165-73.

55. Zimmermann MB, Hurrell RF. Nutritional iron deficiency. Lancet (London, England). 2007:370: 511-20. https://doi.org/10.1016/s0140-6736(07)61235-5.

56. US Departments of Health and Human Services, Centers for Disease Control and Prevention (CDC). Recommendations to prevent and control iron deficiency in the United States. Morbidity and Mortality Weekly Report (MMWR) Recommendations and Reports. 1998:47: 1-29.

57. Department of Veteran Affairs, Department of Defense. VA/DoD Clinical Practice Guideline for Management of Pregnancy. http://www.healthquality. va.gov/guidelines/WH/up/.2009.

58. Guyatt GH, Oxman AD, Ali M, Willan A, Mcllroy W, Patterson C. Laboratory diagnosis of iron-deficiency anemia: an overview. J Gen Intern Med. 1992.

59. Breymann C, Honegger C, Hosli I, Surbek D. Diagnosis and treatment of iron-deficiency anaemia in pregnancy and postpartum. Arch Gynecol Obstet. 2017;296:1229-34. https://doi.org/10.1007/s00404-017-4526-2.

\section{Publisher's Note}

Springer Nature remains neutral with regard to jurisdictional claims in published maps and institutional affiliations. 